\title{
Food waste: The role of date labels, package size, and product category
}

\author{
Norbert L.W. Wilson, Professor \\ Department of Agricultural Economics and Rural Sociology \\ Auburn University \\ Auburn, AL 36849
}

Bradley J. Rickard, Associate Professor (corresponding author)

Charles H. Dyson School of Applied Economics and Management

Cornell University

Ithaca, NY 14853

Tel: +1.607.255.7417

E-mail:bjr83@cornell.edu

Rachel Saputo, Graduate Student

Charles H. Dyson School of Applied Economics and Management

Cornell University

Ithaca, NY 14853

Shuay-Tsyr Ho, Graduate Student

Charles H. Dyson School of Applied Economics and Management

Cornell University

Ithaca, NY 14853

\begin{abstract}
The presence of food waste, and ways to reduce it, has generated significant debate among industry stakeholders, policy makers, and consumer groups around the world. Many have argued that the variety of date labels used by food manufacturers leads to confusion about food quality and food safety among consumers. Here, we develop a between-subject, laboratory experiment with different date labels (Best by, Fresh by, Sell by, and Use by) for products (ready-to-eat cereal, salad greens, and yogurt) of different sizes and dates to evaluate how date labels influence the value of premeditated food waste of subjects, or their willingness to waste (WTW). Subjects have different WTW over products, sizes, and dates; we expect that ambiguity avoidance may prompt differences in the WTW. The WTW is greatest in the "Use by" treatment, the date label which may be the least ambiguous and suggestive of food safety. The WTW is the lowest for the "Sell by" treatment, which may be the most ambiguous date label about safety or quality for the consumer. Results from the mixed-design, repeated measures ANOVA provide evidence that subjects have different WTW by date labels over products.
\end{abstract}

Keywords: Consumer preferences; Date labels; Experimental economics; Food quality; Food safety; Public policy analysis. 


\section{Food waste: The role of date labels, package size, and product category}

\section{Introduction}

The presence of food waste, and ways to reduce food waste, has generated significant debate among industry stakeholders, policy makers, and consumer groups. Arguably, food waste has become one of the top issues for individuals and organizations involved in food marketing and food policy in the United States and elsewhere. The U.N. Conference on Sustainable Development acknowledges food waste and food loss as important components of food insecurity in their Zero Hunger Challenge (Halloran, Clement, Kornum, Bucatariu, \& Magid, 2014). In June 2013, the U.S. Department of Agriculture (USDA) and the U.S. Environmental Protection Agency (EPA) partnered to launch the U.S. Food Waste Challenge, an initiative to reduce food waste throughout the food supply chain. ${ }^{1}$ Some have estimated that annual food waste costs in the United States are approximately $\$ 160$ billion, representing resources that went into the production, distribution, and marketing of food products (Buzby, Wells, \& Hyman, 2014; Newsome et al., 2014, Pierson, Allen, McLaughlin, \& Halloran, 1982). Food waste is also a food security concern as it symbolizes a lost opportunity to feed the 17.4 million food insecure U.S. households (Coleman-Jensen, Rabbitt, Gregory, \& Singh, 2015).

Buzby et al. (2014) estimate that $31 \%$ of food is wasted; this is the total of food wasted by consumers (21\%) and producers (10\%). Given the consumer share of food waste in the United States and several efforts to address food waste in production, distribution, and storage, we work to understand better date labels, such as "Best by”, "Fresh by”, "Sell by”, and "Use by” as a possible avenue to mediate food waste. Specifically, we focus on the effects of different date labels, on anticipated food waste, which may reflect the actual level of food waste. In this analysis we create a new measure of food waste, the willingness to waste (WTW), which is 
based on the value ascribed to the product by subjects and their anticipated wasted. This analysis suggests that date labels may influence consumer willingness to pay (WTP) for food products, the anticipated waste, and the WTW.

A burgeoning literature explores the role of date labels on food waste. Critics argue that date labels are confusing for consumers, and that this confusion encourages unnecessary levels of food waste (Newsome et al., 2014; Wansink \& Wright, 2006; WRAP (Waste \& Resources Action Programme, 2011). Evidence suggests that consumers waste food products as they near the date posted on the date label (open date label) for perceived food safety reasons (Kantor \& Lipton, 1997; Miles \& Frewer, 2001; Newsome et al., 2014; Woodburn \& Van Garde, 1987). In addition to food being discarded for reasons related to perceived food safety, others have shown that consumers waste food for reasons related to food quality (Theotokis, Pramatari, \& Tsiros, 2012; Tsiros \& Heilman, 2005). Wansink and Wright (2006) find that as consumers observe an approaching "Best if Used By" date label, they decrease their acceptance, as well as the perceived healthfulness and freshness of the product.

Despite discussions about the quantity and value of food waste in the United States, little empirical work exists that provides primary data to quantify food waste and describes how food waste may vary across different populations and across different products. Understanding consumer behavior is a key factor in developing a better understanding of the causes of food waste and the consequences of changes that might be employed to mitigate food waste. As part of this discussion, we have witnessed a range of public policy recommendations that have proposed to change the language used on food as it relates to date labels (Newsome et al., 2014). One example is the bill titled "The Food Date Labeling Act” that was introduced in May 2016 in the U.S. House of Representatives and Senate (H.R. 5298 and S. 2947)². 
For the purpose of this research, we developed an experiment to study the factors that influence food waste. We include a range of products to understand how date labels influence anticipated waste for products of increasing perishability. As suggested in the literature, product size and expiration dates may influence waste, so we include products of different sizes and dates to evaluate the effects of these parameters on anticipated waste.

\subsection{Contextual Background}

In the United States, rules about open date labels differ by state, but overall they are widely unregulated. With the exception of infant formula, which is regulated under the 1980 Infant Formula Act, the U.S. Food and Drug Administration (FDA) does not require food products to display specific open (visible) date labels. However, some poultry, meat, and egg products under USDA jurisdiction necessitate date documentation, but phrases including "Sell by" and "Use before” can be used interchangeably (Leib et al., 2013; Newsome et al., 2014). The USDA does not set out strict guidelines for terminology commonly used on food products. The use of the following date label phrases have been summarized by Tsiros and Heilman (2005):

- "Sell By" conveys to the retailer the last date the product can be displayed for sale. It is not an indication of a product's safety or quality.

- “Best if Use By,” "Best Before,” or "Best By” are used to suggest the date after which the food's quality or flavor may deteriorate.

- "Use By" recommends the last date by which the product should be consumed, but does not necessarily convey safety information.

The power to enact additional food date labeling laws rests with sub-national (state and local) authorities, which can include the departments of agriculture, commerce, and health, among other agencies. Additionally, other qualifying phrases such as "Fresh By" or "Enjoy By" 
can be used by food manufacturers. Though neither are officially indicators of a product quality or product safety, they suggest quality. The date labels do have the potential to send signals to consumers and influence preferences (Leib et al., 2013). Labels such as “Best Before” are sometimes perceived to indicate microbial safety rather than freshness, while "Use By" may be interpreted to imply quality, depending on accompanying information. Overall, this lack of jurisdiction by a single agency coupled with manufacturer discretion over the application of date labels has the capacity to foster inconsistencies in terminology and confusion about product safety and quality among consumers (Leib et al., 2013).

This confusion is thought to contribute to the disposal of safe and edible food (Codex Alimentarius Commission, 2014, Newsome et al., 2014; WRAP, 2011). In response to the ambiguity of date labels (date markings), Codex Alimnetarius has a recommendation to consolidate date labels to two: one to indicate safety and one to indicate quality, which is similar to the bills in the U.S. Congress. Evidence also suggests some consumers believe a product past the open expiration date is no longer safe for consumption (Newsome et al., 2014). Van Garde and Woodburn (1987) found that consumers often disposed of food products that were past the open expiration date without additional sensory evaluation. Results from Kantor and Lipton (1997) support this claim; consumers reported not trusting their senses as an accurate judge of a food's edibility, thus preferring to discard food when the quality or safety was questioned. Such evidence implies people place heavier reliance on expiration dates than temperature control, the latter of which is much more important in determining food safety because date labels do not guarantee microbiological food safety (Newsome et al., 2014; Woodburn \& Van Garde, 1987).

Antecedents such as risk perception and experience with food-borne illness may shape perception of and engagement with date labels. Past experiences and the risk a consumer 
associates with a food product also influence how often a person examines the open date label (Tsiros \& Heilman, 2005). Specifically, Kantor and Lipton (1997) found that negative experiences with a food product made consumers more inclined to discard prematurely that product. This increased tendency to waste food because of the expiration date despite lack of apparent safety concerns may be partially attributable to increased consumer awareness and fear of food safety issues (Miles \& Frewer, 2001).

In addition to food products being discarded for perceived safety reasons, a food's quality as signaled by date labels also contributes to food waste. Tsiros and Heilman (2005) found that depending on the product category, between $69 \%$ and $84 \%$ of consumers believe perishable products deteriorate in quality over time. This result is supported by Theotokis et al. (2012), who provide evidence that products priced lower as they near the expiration date prompts consumers to have negative perceptions of brand quality. While the psychological effects of expiration datebased pricing vary among consumers depending on associated risk and brand loyalty, Theotokis et al. (2012) suggest expiration dates influence how consumers perceive the product, and expiration date-based price changes signal inferior quality and ultimately affect consumer purchasing decisions.

Kantor and Lipton (1997) also find that bulk purchases contribute to food waste. Marketing tactics, such as "buy one get one free," may also facilitate impulse purchases, which coupled with poor meal planning, thwarts households from consuming food products before the open date nears (Farr-Wharton et al., 2014). Inadequate storage facilities and practices further contribute to avoidable food waste, as consumers are prone to forgetting or miscalculating what food they currently have in stock (Kantor \& Lipton, 1997). This culture of abundance and reliance on date labels may induce consumers to dispose of food products past the open date 
label (Godfray et al., 2010). Through the use of an economic experiment that assesses responses to different date labels across products, package sizes, and dates, we hope to provide new insights into how date labels may shape anticipated food waste.

\section{Theory and Hypotheses}

As suggested in the literature, consumers may discard products based on the expiration date because of concerns of safety and quality even without other forms of verification. The date labels and the expiration dates may also influence perceptions of quality. As a result, perceptions of quality and safety may inform consumers' expected consumption levels (and therefore their expected amount of food waste) at the point of purchase. That is, based on the date label and the expiration date, consumers may knowingly purchase a product with the idea that some portion of it will not be consumed, i.e., some of the value of the product will be discarded. The expected amount of food waste at the time of purchase could then be moderated by consumption patterns and influences in the home. As date labels influence perceptions of quality and safety, we argue that date labels and the expiration dates will influence consumers' value of the product and their expected amount of consumption (and waste); we refer to this expected amount of food waste as premeditated food waste $(\mathrm{PW})^{3}$. The existence of PW highlights that consumers implicitly place some economic value on the option to waste food for reasons related to convenience, safety, uncertainty, etc., and this motivates us to construct a measure that appropriately quantifies the value of PW.

Here we develop a measure of the value of PW that we refer to as the WTW; the WTW is based on the information we collect from subjects that describe their WTP for each item and the amount of the item they expect to consume $e^{4}$. The expected consumption level ranges from $0 \%$ to $100 \%$; we subtracted this value from $100 \%$ to attain the PW for each item. We then multiplied 
the PW times the maximum WTP for each food item for each subject. This WTW measure follows a concept that has been employed in the nutrition literature (e.g., Woodburn \& Van Garde, 1987) that measures the “cost of discarded food” as a measure of food waste.

By construction, WTW will adjust to changes in WTP and PW. We assert that at the point of purchase consumers have a WTP and a level of PW for the product, and these factors interact. That is, perception of the date label may influence valuation of the product and PW simultaneously. In the experimental design, we use the relationship between PW and WTP in the auction by asking subjects to report their PW and WTP concurrently.

A few important caveats are necessary: The planning fallacy as discussed by Kahneman and Tversky (1979) and Kahneman (2011) suggests that people are prone to be overly optimistic about future plans or they expect to repeat previous outcomes. Thus asking subjects to predict future consumption may be biased upward thus biasing down their PW and WTW. Our assumption is that if the bias exists, it is consistent across all subjects and treatments. While the cost of discarded food has been used before, WTW is a new construct with no benchmarks or external validity checks. For all of these issues, we do not look at the WTW or PW as absolute values. Rather, the point of this analysis is to study the relative effects of the date labels on WTW. If date labels cause differences in the WTW, and its constituents, then we have evidence that some labels may prompt consumers to accept a greater cost of waste.

Based on the literature, we expect that consumers will bear the cost of waste based on the date labels regardless of the intent or purpose of the date label. Even at the point of purchase we expect that a large share of customers will accept some cost of waste (Farr-Wharton et al., 2014); that is, consumers have a baseline WTW regardless of the product features (date, size or shelf life) and the date label. We are careful to claim that our experimentally derived WTW is only 
one potential estimate of the actual cost of food waste. However, we expect a consistent response between the actual cost of waste and the WTW over product features by date labels.

\subsection{Hypotheses}

Since WTW is the product of WTP and PW, we develop a series of hypotheses which will help in the construction of the hypotheses of WTW. To assure that subjects responded in the auction in a theoretically consistent manner, we start with a series of hypotheses related to consumer response to product (shelf life), date, and size.

\subsubsection{Hypotheses for Product, Date, and Size}

We hypothesize that products that have a shorter shelf life, e.g., salad, will generate greater PW than products that have a longer shelf life, e.g., cereal (Theotokis et al., 2012, cf. Wansink \& Wright, 2006). We hypothesize that PW is greater for expiration dates that are nearer compared to farther. The larger versions of the products will generate greater PW than smaller versions (Kantor \& Lipton, 1997). We hypothesize that these relationships hold for each date label, that is the date label does not disrupt the hypothesized relationships across products, dates, and sizes.

$$
\begin{gathered}
H_{1}: P W_{\text {Shorter Shelf Life }}>P W_{\text {Longer Shelf Life }} \\
H_{2}: P W_{\text {Nearer Date }}>P W_{\text {Farther Date }} \\
H_{3}: P W_{\text {Large }}>P W_{\text {Small }}
\end{gathered}
$$

Subjects should be willing to pay more for a product with a farther expiration date than products with a nearer date, as the market has examples of discounts for nearly expired products. Economic reasoning suggests that subjects should be willing to pay more for larger products than smaller products. These relationships should hold over date labels. That is, the pattern of the WTP by dates and sizes should hold regardless of date label.

$$
H_{4}: W T P_{\text {Nearer Date }}<W T P_{\text {Farther Date }}
$$




$$
H_{5}: W T P_{\text {Large }}>W T P_{\text {Small }}
$$

We do not have a hypothesis of the rank order of the WTP by product, as other economic factors may shape how subjects value a product with a shorter shelf life versus a different product with a longer shelf life. However, across these products, we expect $H_{4}$ and $H_{5}$ to hold.

As a final check of internal validity, we hypothesize that WTW will vary by product, date, and size. Thus, subjects have a higher WTW for products with a shorter shelf life. The difference in WTW will vary by product size and date such that products with a closer expiration date will have a higher WTW than products with a later expiration date and larger products will have a higher WTW than smaller products.

$$
\begin{gathered}
H_{6}: W T W_{\text {Shorter Shelf Life }}>W T W_{\text {Longer Shelf Life }} \\
H_{7}: W T W_{\text {Nearer Date }}>W T W_{\text {Farther Date }} \\
H_{8}: W T W_{\text {Large }}>W T W_{\text {Small }}
\end{gathered}
$$

\subsubsection{Hypotheses of the Date Label Effects}

We hypothesize that the constituents of WTW (WTP and PW) are statistically different by date label. The conditions in our experiment are the date labels ("Best by", "Fresh by", "Sell by", and "Use by") most commonly found in the market. Based on the literature on ambiguity aversion, we assert that the differences in effects are based on the ambiguity of the date labels (Ellsberg, 1961; Fox \& Tversky, 1995; Goldberg, Roosen, \& Nayga, 2009; Kivi \& Shogren, 2010). We assert that "Sell by" is ambiguous and the least restrictive of the date labels of the four that we study. Consumers perceive the "Sell by" date label to have a wide range of meanings (safety, quality, message to retailer, etc.) and it is inherently less clear than "Use by", 
which suggests safety, and "Best by" or "Fresh by", which suggest quality. Safety, at least in the food realm, is inherently less ambiguous than quality. Thus, a label suggesting safety may suggest greater risk of food-borne illnesses, while quality labels are related to many different features such as flavor profile, freshness, color, and safety, among others. In addition, some of these features are subjective, and this exacerbates the ambiguity of quality relative to safety.

In terms of quality, the "Fresh by" date label is the clearest statement of product quality, as the label is indicative of a particular quality characteristic. "Best by" may also be considered to be a quality signal, but we argue that it is a more ambiguous signal than the "Fresh by" date label. Therefore, we hypothesize that PW is higher for date labels that are ambiguous and about unspecified quality, namely "Sell by", and lower for date labels that are clearer and more about safety, namely "Use by". As a result, we hypothesize that PW will increase by date labels in the following order: "Use by”, "Fresh by”, "Best by”, and "Sell by”.

$$
H_{9}: P W_{\text {Clearer Date Label }}<P W_{\text {More Ambiguous Date Label }}
$$

Because consumers want to avoid ambiguity, we hypothesize that WTP is greater for date labels that are suggestive of safety (clearer) over date labels that suggest quality (more ambiguous). Similar to Goldberg et al. (2009) and Kivi and Shogren (2010) who found evidence of a higher WTP to avoid ambiguity, we hypothesize that "Use by" leads to a higher WTP over more ambiguous date labels or date labels about quality. We hypothesize that WTP is lower for date labels that are more ambiguous and more about unspecified measures of quality. For example, "Sell by" leads to a lower WTP relative to date labels that are clearer and more about safety. Thus, the WTP declines by date labels following this order: "Use by”, "Fresh by”, "Best by", and "Sell by".

$$
H_{10}: W T P_{\text {Clearer Date Label }}>W T P_{\text {More Ambiguous Date Label }}
$$


Economic reasoning suggests that a negative relationship may exist between PW and WTP, which suggests that consumers make trade-offs in value and waste. If consumers adjust WTP (PW) more to date labels than PW (WTP), then WTW will follow WTP (PW). However if consumers make near equal adjustments between WTP and PW by date labels, no effect will be observed in the WTW. We argue that WTP falls as the date labels become more ambiguous, and we assert that the PW rises with ambiguity but at a slower rate. That is, we assert that ambiguity aversion has a stronger effect on WTP than on PW. As a result, consumers are more willing to bear a higher cost of PW when the date label is clearer about safety and are less willing to bear the cost of PW when the date label is ambiguous about quality. Thus, we hypothesize that "Use by" generates the highest WTW while the "Sell by" date label generates the lowest WTW of the date labels. We hypothesize that the WTW will follow in descending order "Use by", "Fresh by", "Best by", and "Sell by”. This ranking will hold across all products, sizes, and dates.

$$
H_{11}: W T W_{\text {Clearer Date Label }}>W T W_{\text {More Ambiguous Date Label }}
$$

\section{Material and methods}

\subsection{Collecting data to measure food waste}

To study the effect of date labels on food waste, and to understand better the implications of date labels on food waste, we need to assess critically an estimate of food waste. A wide range of estimates for food waste exist in the United States, and much of the range is attributable to the methods employed to calculate the quantity and/or value of food wasted. Measuring food waste could be done using food recall surveys or by observing individuals in a public setting. Food waste might also be measured as part of a consumer-based experiment, and we argue that a properly designed experiment might be a more ideal method to understand current levels of food waste and the effects of tools that might influence food waste. 
Experimental economists have established credible methods to collect price data that are incentive compatible—see Lusk and Hudson (2004) and Lusk, Fields and Prevatt (2008) for a nice summary of the most common experimental designs used by applied economists. Little research in the economic literature exists that uses experiments to collect consumption data, and no earlier work that uses experimental methods to collect data on food waste.

\subsection{The auction}

We designed an experiment that allowed us to collect data to study the drivers of food waste, and the role of date labels on food waste, among consumers. Data were collected from 200 nonstudent subjects that participated in our laboratory experiment. Subjects were randomly placed in one of four conditions differentiated by the date label ("Best by", "Fresh by", "Use by" or "Sell by"). We ran multiple sessions of each date label—-three sessions for "Best by" and two sessions for the other date labels—and each session consisted of between 16 and 25 subjects. For each food item, subjects were asked to place their WTP bid and indicate the percentage of the item that they expect that their household will consume. At the end of the auction rounds all subjects completed a short computerized survey with questions related to demographic variables and food consumption habits.

Subjects recorded their WTP and the percentage of the product they expected to consume for each food item presented to them in the auction. In the experiment subjects evaluated three products (yogurt, ready-to-eat breakfast cereal, and salad greens) in two sizes (small and large): yogurt products (5.3 ounce (150.3 grams) and 32 ounce (907.2 grams)), two ready-to-eat cereal products (10 ounce (283.5 grams) and 40.7 ounce (1.15 kilograms)), and two pre-washed salad green products (5 ounce (141.7 grams) and 10 ounce (283.5 grams)). For each size of product, subjects evaluated three different expiration dates (near, middle, and far) giving 
a total of 18 items total (3 products X 2 sizes X 3 dates). The dates of the products varied by near, middle and far dates. The dates varied by product to reflect commonly found expiration dates in the market (cereal: 1 month, 3 months, and 1 year; salad: 1 day, 3 days and 1 week; and yogurt: 1 day, 3 weeks, and 1 month). All dates were presented as MM/DD/YYYY.

As a between subject experiment, subjects saw only one date label (“Best by”, "Fresh by”, "Sell by” or "Use by”), and the date label was constant throughout the session. Further, each subject saw the same 18 items. Thus the experiment is a mixed design with repeated measures. To limit ordering effects, we randomly altered the sequence of the presentation of the products across sessions.

For all sessions, we used the Becker-DeGroot-Marschak (BDM) auction to elicit bids for the items from all subjects. Given that subjects may have a wide range of valuations, the BDM is an ideal elicitation method because subjects do not bid against each other. Rather, they submit a sealed bid for each item and then have the chance to "win” a product if their bid exceeds a randomly drawn price. After all bids were submitted in a session, we randomly chose a market price for a selected item.

\section{Results}

Our sample contains 3600 observations; this includes the information submitted from 200 subjects on the 18 different food items. Table 1 presents the summary statistics for the three outcome variables PW, WTP, and WTW for each date label. We conducted the analysis with Stata 14.1 SE.

\subsection{Summary Statistics}

The results in Table 1 show that PW is smallest for "Use by" consistent with $H_{9}$. PW is largest for "Best by", which is inconsistent with the hypothesis. The general order of the PW follows the 
hypothesis; however, the confidence intervals overlap suggesting no statistical differences. For WTP, "Use by" is associated with the largest values in support of $H_{10}$. The lowest WTP is from the "Best by" treatment, which is inconsistent with $H_{10}$. By overlapping confidence intervals, "Best by" and "Sell by" are not statistically different. Nevertheless, the general ranking is as hypothesized. The WTW follows $H_{11}$ as WTW is largest for "Use by" and smallest for "Sell by". ${ }^{5}$ However, overlapping confidence intervals indicate that "Use by" is not statistically different than "Fresh by", and "Best by" is not statistically different than "Sell by". However, the pairs are statistically different $(p<0.05)$.

PW and WTP by date label have a negative correlation $(\rho=-0.42)$. Thus, the results support the hypothesized negative relationship between WTP and PW. Further, regression analysis suggests that PW and WTP may be contemporaneously determined (which is available upon request). That is subjects that value a product more may state that they will waste less of the product. Thus, the simultaneity of the decision to purchase and to consume (or waste) encouraged us to create the WTW, and to employ this measure in our analysis as the most appropriate measure of the value of food waste across the food items studied. Further exploration of this simultaneity is beyond the scope of this paper, as the main goal here is to assess if the different date labels lead to different WTW given the simultaneity embedded. As mentioned in the explanation of $H_{11}$, WTW follows WTP more that PW does. In fact, the correlation coefficient between WTP and WTW is relatively high $(\rho=0.56)$ and the correlation coefficient between PW and WTW is lower $(\rho=0.22)$ suggesting that WTP may be the dominant factor in the WTW measure. 


\subsection{Mixed-Design ANOVA with Repeated Measures}

We turn to a mixed-design ANOVA with repeated measures in Table 2. The model that we run consists of the outcome variables (PW, WTP, and WTW) and the independent variables are the Date label (“Best by”, “Fresh by”, "Sell by”, and “Use by”), Product (cereal, salad, and yogurt), Date (far, middle, and near), and Size (small and large). As suggested by the hypotheses, we are interested initially in how the outcome variables change by product, date, and size. Then we explore the effect of date labels on the outcome variables. Finally, we assess the effects of date labels across different products, dates, and sizes. Thus in the models, we incorporate interactions of date labels with each repeated measure of product, date, and size separately. Each subject is exposed to only one date label, therefore we nest subjects within date labels. We then recognize this in the error structure of the date label. We also acknowledge the repeated measures of product, date, and size in the model specification.

In our mixed-design ANOVA model, we find that Product, Date, and Size are statistically significant for each outcome variable $(p<0.001)$, which provides support to $H_{1}, H_{2}, H_{3}, H_{4}, H_{5}, H_{6}, H_{7}$, and $H_{8}$ (Table 2). We find that the direct effect of Date Label is not statistically significant for each outcome variable, which does not provide direct support of $H_{9}, H_{10}$, and $H_{11}$. However, we find that the interaction term of Date Label and Product is statistically significant for $\operatorname{WTP}(p<0.01)$ and WTW $(p<0.001)$, which suggest that $H_{10}$ and $H_{11}$ may hold for certain products. We do not find a statistically significant interaction of the Date Label with Date or Size, which support the hypotheses that the responses to Date and Size are not affected by the Date Label. 


\subsubsection{Results for Direct Effects}

We provide the ANOVA contrasts for the three outcome variables for the direct effects, which confirm nearly all of the hypotheses related to Product, Date, and Size. In Table 3, if the estimated margins, upon which the contrasts are based, share a letter in the group label (connecting letters), then the contrasts are not statistically significant at the 5\% level. In the case where no connecting letter is present, the margin and all of its contrasts are statistically different $(p<0.05)$

The hypotheses $H_{1}$ and $H_{6}$ state that products with a shorter shelf life relative to those with a longer shelf life will have a larger PW and smaller WTW. Each ANOVA contrast for PW and WTP is statistically different; thus, no connecting letters are presented. While the ordering of the hypothesis holds for salad relative to yogurt, cereal, which is the least perishable product, has the second highest PW and the highest WTW. Interestingly, the WTP for cereal is statistically different than salad and yogurt, which are not statistically different.

By date we see evidence that support $H_{2}, H_{4}$, and $H_{7}$, which state that for far dates relative to near dates subjects expect to have lower PW and WTW, and higher WTP. The PW for each date is statistically different from each other and in the hypothesized order. Similarly, the WTP is statistically different for farther dates and follows the hypothesized order. The WTW is lowest for the far date and statistically different than the other date labels, but the middle and near dates are not statistically different.

For the sizes, large products relative to small products yield higher PW, WTP, and WTW, which is consistent with $H_{3}, H_{5}$, and $H_{8}$. The differences by outcome variables are statistically significant and follow the hypothesized order. These results support the first eight hypotheses, and they suggest that subjects responded to the auction in a theoretically consistent manner. 


\subsubsection{Results for the Interaction Effects}

In Table 4, we report the ANOVA contrasts for PW, WTP, and WTW for the interaction of Date Label and Product. For cereal, the ANOVA contrasts indicates that the PW differs by date label such that "Use by" has the smallest PW compared to "Fresh by" and "Best by", though it is not statistically different than "Sell by". This result is generally consistent with $H_{9}$. However, cereal is the only product that supports this hypothesis. PW is not statistically different for salad and yogurt, by date label. This result is inconsistent with $H_{9}$. Across the products, we see that subjects report higher PW for salad, the product with the shortest shelf life, relative to cereal and yogurt $($ contrast $=5.57, p<0.001$ and contrast $=9.31, p<0.001)$, which is consistent with $H_{1}$.

However, the PW for cereal is larger than the PW for yogurt (contrast $=3.74, p<0.01$ ), which is inconsistent with $H_{1}$.

We find evidence that the date labels lead to statistically different WTP within and across products. In line with $H_{10}$, "Use by", the most clear date label, generates a larger WTP than the more ambiguous date labels for cereal and salad. While the WTP for cereal with "Use by" is larger than the other date labels, consistent with the order of $H_{10}$ " "Sell by" has a larger WTP than the WTP for "Best by", contrary to $H_{10}$. For salad, the WTP with "Use by" is statistically different than the WTP for other date labels. However, the WTP for the other date labels are not statistically different. For yogurt, "Use by” and "Fresh by" are not statistically different. The WTP for "Fresh by" is not statistically different than the WTP for "Best by" or "Sell by", which is not consistent with the order of $H_{10}$.

The results by date label for WTP and PW suggest limited statistical differences across date labels, particularly for "Best by”, "Fresh by” and "Sell by”. Yet the results suggest WTP 
and PW respond differentially for "Use by" relative to the other date labels. This difference suggests that the WTW may have statistically different effects by date label. As seen with WTP and PW in Table 2, the direct effect of Date Label on WTW, given the mixed-design, is not statistically significant $(p=0.14)$. However the interaction of Date Label and Product is statistically significant $(p<0.001)$. This result suggests that $H_{11}$ does not hold in general, but for certain products, this hypothesis may still hold.

For salad and yogurt, "Use by" has the highest WTW; however, the WTW for cereal is lower that the WTW for "Use by" and "Fresh by", but they are not statistically different from each other. The general order of $H_{11}$ is supported: "Use by" and Fresh by" lead to higher WTW for cereal than "Best by" and "Sell by". For salad, "Use by" generates a WTW that is greater than the WTW for all other date labels, "Fresh by" leads to a greater WTW than "Sell by". Similarly, for yogurt, "Use by" has a higher WTW than the other date labels, but all the other date labels are not statistically different.

Across the products, cereal, which has the longest shelf life, has the highest WTW over the other products (cereal versus salad contrast $=0.14, p<0.001$ and for cereal versus yogurt contrast $=0.27, p<0.001)$. The result is counter to $H_{6}$. However, by product, we see that salad, the most perishable product, with the date label "Use by" has the highest WTW compared to all other products and date labels (though not statistically different than "Fresh by" for cereal). This result supports $H_{6}$ at least by date label. Further support to $H_{6}$ is that salad has a higher WTW than yogurt ( contrast $=0.13, p<0.001)$. By date label, salad with "Fresh by" or "Best by" has a higher WTW than yogurt with "Best by" and "Sell by". 


\section{Discussion}

Food waste has become one of the top food policy issues in the United States and elsewhere. Because much of the food waste in the United States happens at the consumer level, it is particularly important to understand better consumer attitudes and behaviors regarding food consumption and food waste. In addition, it is important to understand the likely reaction of consumers to policy initiatives used to mitigate food waste. Earlier work has developed measures to quantify the level of food waste by consumers, and some research has been done that begins to assess how consumers make decisions that affect the amount of food that is wasted (for a nice summary see Neff, Spiker, and Truant 2015).

Thus earlier work motivated our hypothesis that different language on date labels would differentially affect the value of food wasted by consumers. Some date labels are purported to influence consumer perception of food and beverage products by making implicit references to either the quality or the safety of the product. It is difficult to properly test this hypothesis using store-level data or data from a survey instrument. However, by developing a controlled laboratory experiment we are able to test this hypothesis directly. Our results clearly show that, holding other factors constant, date labels do impact consumer behavior and the value of the food that they intend to waste. This finding supports work that suggests that consumers have concerns of product safety and have a lower perception of the quality once the product is beyond the date label (Kantor \& Lipton, 1997; Newsome, et al., 2004; Theotokis et al. 2012; WRAP, 2011; Woodburn \& Van Garde, 1987). Overall, our results suggest that the debate about regulating the manufacturers' use of date labels is not misguided, and that there may be a role for policy that tries to regulate or harmonize the date label language used on food and beverage products. 
Harmonizing the date labels may lead to lower WTW, but the response to the reduction will vary by product. Across the three products, we see differential responses to the date labels. Subjects have a WTW that is nearly 50\% larger for "Use by" and "Fresh by" relative to "Best by" and "Sell by". In contrast the WTW for "Use by" for salad is nearly three times greater than "Sell by". However harmonizing the date labels to "Best by" or "Sell by" may have unintended consequences including effects on consumers' WTP. Further, as suggested by the ambiguity of date labels, "Best by" and "Sell by" provide consumers little information. Thus harmonizing to these date labels may lead to effects beyond what can be assessed in our experiment.

Since "Use by" yields the greatest WTW under nearly every condition, "Use by" should be adopted in limited ways to express real threats to safety for perishable products, a recommendation similar to Codex Alimentarius and the two bills before the U.S. Congress. Even though "Sell by" generates the lowest WTW, this date label may be best as a closed date label (not shown to consumers) as it conveys little information to consumers. For less perishable products, a date label about the specific quality characteristics of the product may convey better information, but to prevent the challenges mentioned, a clear education campaign is necessary to explain the meaning of a new unified label.

Further, our hypotheses are based more on the ambiguity of language used in the date label, though we do not formally test ambiguity. As part of the on-going policy discussions about ways to mitigate food waste, we see proposals that support (or advocate against) specific language in the date label. In the experiment we used four date labels that each used different language. One date label was more suggestive of a food safety concern ("Use by”), two were more suggestive of a food quality concern ("Fresh by" and "Best by"), and one was suggestive of retailer responsibilities rather than a label directed at the final consumer ("Sell by”). The 
overarching finding is that a date label about safety ("Use by") may trigger the acceptance of a greater cost of food waste (WTW) relative to date labels about quality or other attributes ("Best by”, "Fresh by” or “Sell by”).

Finally, our hypotheses consider the effects of product, date, and size. Here we expected that subjects would have a larger response to date labels for the most perishable products, for the dates that were closest to the purchase date, and for larger product sizes. Similar to Kantor and Lipton (1997) and Farr-Wharton et al. (2014), our results suggest that consumers may adjust their PW, WTP, and WTW by product features. Since the impact of expiration date and package size on food waste is a non-trivial concern, these impacts need to be examined more closely in subsequent research to understand more fully how the use of date labels affects food waste over time and across package sizing.

For example, states that make the sale of products beyond the expiration date illegal, e.g. milk in Montana (Leib et al. 2013), may lead to greater waste when the product may be fine beyond that specific date. This result suggests that varying product size may be a possible market solution to food waste. Subjects anticipated wasting (PW and WTW) more when presented larger package sizes. While consumers may receive a quantity discount for larger sizes, this saving may be lost through waste. The potential environmental cost of more packaging is an issue worth considering. Nevertheless, further exploration of expiration dates and package size may be a tool that industry can use to combat food waste.

Beyond the effect of date labels on the value of PW, we find evidence of a tradeoff in the value versus the quantity of waste. The negative correlation between WTP and PW suggests that, even at the point of purchase, consumers may make a determination of their perceived value of the product and future consumption. The finding suggests that consumers are potentially less 
likely to waste if they value the product more. Thus efforts to remind consumers of the value of products may shift consumers' PW, which may shift realized waste.

Concerning method, we used a between subject experiment structure. While this allowed for subjects to focus on only one date label, we are not able to evaluate how the same subject considered the different date labels. This structure may have contributed to the differential effects of date labels across products. However as noted in the literature, subjects may not have had a clear sense of the differences in the date labels. We cannot distinguish these causes given the experimental design. Despite this issue, our results indicate date labels influenced consumer perception of the products.

Our analysis contributes to the literature in multiple ways, notably with the introduction of PW and WTW. Measuring food waste is challenging: self-reporting has the potential for bias to avoid looking like a "food waster". Weighing plate waste has become a popular method to evaluate the quantity of food waste, but this does not tell us how subjects may respond to characteristics of the product in the store or at home. While not a perfect measure, asking subjects the amount of product that they expect to consume yielded a unique method to measure future waste. These two metrics may permit future researchers innovative ways to study food waste in an experimental setting.

\section{Conclusion}

Researchers, policy makers, and consumers are questioning the efficacy of various mechanisms and policies that might be used to reduce food waste, but little economic research exists that examines the alternatives carefully. Our research uses data collected in an incentive compatible experiment to assess how consumers respond to different date labels for food products in small 
and large package sizes. We find that the value of consumer food waste does respond to date labels, and that the effects vary across food categories.

Our findings show that a date label that is most suggestive of a food safety concern leads to the greatest value of food wasted. The idea that food waste is linked to concerns about food safety has support in the literature (Kantor \& Lipton, 1997; Miles \& Frewer, 2001; Tsiros \& Heilman, 2005). The date labels that are more suggestive of a food quality concern lead to less value of food wasted by subjects in our experiment. Nevertheless, some waste occurred regardless of date label as suggested by Theotokis et al. (2012). Results from our experiment provide additional support for policy proposals that seek to regulate date labels on food and beverage products. No well-defined rules exist that differentiate the use of the various date labels that we observe in the marketplace, yet our results highlight that date labels affect the decisions about food waste. Consumers in our experiment anticipated wasting a higher value of food when they were presented with the "Use by" date label, and this result was statistically significant. Policies that look to re-evaluate how food and beverage products are labeled may be useful as part of a larger regulatory effort to reduce food waste (See Leib et al., (2013), for a number of recommendations). As part of this discussion, regulators should consider the full economic and food waste implications of policies that might lead to the harmonization of date label language across all food and beverage products.

Our research, by necessity, focused on a subset of food products sold at retail markets, but we believe that our results shed light on the issue of food waste more generally. Furthermore, we introduce a carefully designed experimental auction that allowed us to control many aspects of consumer choice for the selected food products, and we present a novel method to calculate the value of PW by consumers. Our framework can be extended to examine a wide 
range of issues related to food waste, and the results can be then used to investigate the effects of specific policies designed to mitigate food waste. 


\section{Acknowledgements}

The project was supported by the Agricultural and Food Research Initiative Competitive Program of the USDA National Institute of Food and Agriculture (NIFA), grant number 201467024-21849 and through Hatch project NYC-121864; Multistate S1050. Any opinions, findings, conclusions, or recommendations expressed in this publication are those of the authors and do not necessarily reflect the view of the National Institute of Food and Agriculture (NIFA) or the United States Department of Agriculture (USDA). Additional support is provided by the Alabama Agricultural Experiment Station at Auburn University.

\section{Endnotes}

${ }^{1}$ A summary of the USDA report can be found at (http://www.usda.gov/oce/foodwaste/usda_commitments.html).

${ }^{2}$ Additional details for this bill can be found at: https://pingree.house.gov/media-center/pressreleases/introducing-commonsense-bill-standardaize-food-date-labelng

${ }^{3}$ We thank Brian Wansink for suggesting we consider using the term "premeditated waste”.

${ }^{4}$ It is widely considered that asking people directly the amount of PW they expect to produce will lead to an underestimate of food waste because of the negative connotations associated with food waste.

${ }^{5}$ The mean WTW across date labels shown in Table 1 is not the mean WTP multiplied by the mean PW. Individual WTW measures (that are used to calculate the mean WTW by date label) will be zero when either their WTP or their PW is equal to zero. For an individual, a zero PW linked to a positive WTP will yield a zero WTW value, and a zero WTP linked to a positive PW will also yield a zero WTW value. 
Table 1. Date Label Effects on Mean Premeditated Waste (PW), Willingness to Pay (WTP), and Willingness to Waste (WTW)

\begin{tabular}{llllccc}
\hline & & Use By & Fresh By & Best By & $\begin{array}{c}\text { Sell By } \\
\text { Over All Date } \\
\text { Labels }\end{array}$ \\
\hline PW as a percentage & M & 42.065 & 42.59 & 45.60 & 44.74 & 43.99 \\
(100\%-Anticipated & SD & 1.51 & 1.42 & 1.18 & 1.43 & 0.68 \\
Consumption \%) & 95\% CI & $39.11,45.022$ & $39.81,45.37$ & $43.29,47.91$ & $41.93,47.55$ & $42.65,45.33$ \\
& M & 1.95 & 1.57 & 1.44 & 1.52 & 1.59 \\
WTP in dollars & SD & 0.081 & 0.052 & 0.040 & 0.056 & 0.278 \\
& 95\% CI & $1.79,2.11$ & $1.47,1.68$ & $1.36,1.52$ & $1.41,1.63$ & $1.54,1.65$ \\
WTW in dollars & M & 0.60 & 0.46 & 0.34 & 0.30 & 0.41 \\
WTW=WTP*PW & SD & 0.059 & 0.030 & 0.016 & 0.020 & 0.016 \\
& 95\% CI & $0.48,0.72$ & $0.40,0.52$ & $0.31,0.38$ & $0.26,0.34$ & $0.38,0.44$ \\
\hline
\end{tabular}


Table 2. Mixed-Design ANOVA with Repeated Measures of Product, Date, and Size with Between-Subject Error Term based on Subjects Nested in Date Labels

\begin{tabular}{|c|c|c|c|c|c|c|}
\hline \multirow[b]{2}{*}{ Variable $(d f)$} & \multicolumn{2}{|c|}{ PW in dollars } & \multicolumn{2}{|c|}{ WTP in percentage } & \multicolumn{2}{|c|}{ WTW in dollars } \\
\hline & F-stat & $p$ & F-stat & $p$ & F-stat & $p$ \\
\hline Product $(2,3599)$ & 27.66 & 0.000 & 253.16 & 0.000 & 53.84 & 0.000 \\
\hline Date (2, 3599) & 319.40 & 0.000 & 224.84 & 0.000 & 14.63 & 0.000 \\
\hline Size $(1,3599)$ & 139.95 & 0.000 & 529.57 & 0.000 & 202.66 & 0.000 \\
\hline Date Label $(3,3599)$ & 0.26 & 0.86 & 2.14 & 0.097 & 1.83 & 0.14 \\
\hline Product X Date Label ${ }^{\mathrm{a}}(6,3599)$ & 1.84 & 0.14 & 3.22 & 0.0037 & 6.29 & 0.000 \\
\hline Date X Date Label ${ }^{\mathrm{a}}(6,3599)$ & 0.64 & 0.59 & 0.95 & 0.46 & 1.33 & 0.24 \\
\hline Size X Date Label ${ }^{\mathrm{a}}(3,3599)$ & 0.23 & 0.87 & 0.36 & 0.78 & 1.91 & 0.13 \\
\hline
\end{tabular}

${ }^{a}$ The $F$-stat for the interaction terms are based on the estimates of the respective repeated variable. 
Table 3. ANOVA Contrasts of Direct Effects for Premeditated Waste (PW), Willingness to Pay (WTP), and Willingness to Waste (WTW) from the Mixed-Design ANOVA with Repeated Measures of Food, Date, and Size with Between-Subject Error Term based on Subjects Nested in Date Labels

\begin{tabular}{|c|c|c|c|c|c|c|}
\hline & \multicolumn{2}{|c|}{ PW in percent } & \multicolumn{2}{|c|}{ WTP in dollars } & \multicolumn{2}{|c|}{ WTW in dollars } \\
\hline & $\begin{array}{l}\text { Margin } \\
95 \% \text { CI }\end{array}$ & $\begin{array}{l}\text { Connecting } \\
\text { Letters }\end{array}$ & $\begin{array}{l}\text { Margin } \\
95 \% \text { CI }\end{array}$ & $\begin{array}{l}\text { Connecting } \\
\text { Letters }\end{array}$ & $\begin{array}{l}\text { Margin } \\
95 \% \text { CI }\end{array}$ & $\begin{array}{l}\text { Connecting } \\
\text { Letters }\end{array}$ \\
\hline & \multicolumn{6}{|c|}{ Product } \\
\hline Cereal & $\begin{array}{c}43.38 \\
41.64,45.12\end{array}$ & & $\begin{array}{c}2.18 \\
2.12,2.25\end{array}$ & & $\begin{array}{c}0.55 \\
0.51,0.59\end{array}$ & \\
\hline Salad & $\begin{array}{c}48.95 \\
47.21,50.69\end{array}$ & & $\begin{array}{c}1.33 \\
1.27,1.40\end{array}$ & A & $\begin{array}{c}0.40 \\
0.37,0.45\end{array}$ & \\
\hline \multirow[t]{2}{*}{ Yogurt } & $\begin{array}{c}39.65 \\
37.90,41.39 \\
\end{array}$ & & $\begin{array}{c}1.26 \\
1.20,1.33\end{array}$ & A & $\begin{array}{c}0.28 \\
0.24,0.31 \\
\end{array}$ & \\
\hline & \multicolumn{6}{|c|}{ Date } \\
\hline Far & $\begin{array}{c}29.29 \\
27.54,31.029\end{array}$ & & $\begin{array}{c}2.034 \\
1.97,2.097\end{array}$ & & $\begin{array}{c}0.33 \\
0.30,0.37\end{array}$ & \\
\hline Middle & $\begin{array}{c}41.50 \\
39.76,43.24\end{array}$ & & $\begin{array}{c}1.67 \\
1.61,1.73\end{array}$ & & $\begin{array}{c}0.47 \\
0.43,0.50\end{array}$ & A \\
\hline \multirow[t]{2}{*}{ Near } & $\begin{array}{c}61.19 \\
59.45,62.94\end{array}$ & & $\begin{array}{c}1.075 \\
1.012,1.14\end{array}$ & & $\begin{array}{c}0.47 \\
0.40,0.47\end{array}$ & A \\
\hline & \multicolumn{6}{|c|}{ Size } \\
\hline Large & $\begin{array}{c}50.094 \\
48.67,51.52\end{array}$ & & $\begin{array}{c}2.024 \\
1.97,2.075\end{array}$ & & $\begin{array}{c}0.56 \\
0.43,0.59\end{array}$ & \\
\hline Small & $\begin{array}{c}37.89 \\
36.47,39.31 \\
\end{array}$ & & $\begin{array}{c}1.16 \\
1.11,1.22\end{array}$ & & $\begin{array}{c}0.26 \\
0.23,0.29\end{array}$ & \\
\hline
\end{tabular}

Note: Margins sharing a letter in the group label are not significantly different at the $5 \%$ level. The relevant comparison for the connecting letters is within Product, Date or Size for PW, WTP, or WTW separately. The ANOVA contrasts are not corrected for multiplicity and empty cells are rebalanced. 
Table 4. ANOVA Contrasts of Interacted Effects for Premeditated Waste (PW), Willingness to Pay (WTP), and Willingness to Waste (WTW) from the Mixed-Design ANOVA with Repeated Measures of Food, Date, and Size with Between-Subject Error Term based on Subjects Nested in Date Labels

\begin{tabular}{|c|c|c|c|c|c|c|}
\hline & \multicolumn{2}{|c|}{ PW in percent } & \multicolumn{2}{|c|}{ WTP in dollars } & \multicolumn{2}{|c|}{ WTW in dollars } \\
\hline & $\begin{array}{l}\text { Margin } \\
95 \% \text { CI }\end{array}$ & $\begin{array}{l}\text { Connecting } \\
\text { Letters }\end{array}$ & $\begin{array}{l}\text { Margin } \\
95 \% \text { CI }\end{array}$ & $\begin{array}{l}\text { Connecting } \\
\text { Letters }\end{array}$ & $\begin{array}{l}\text { Margin } \\
95 \% \text { CI }\end{array}$ & $\begin{array}{l}\text { Connecting } \\
\text { Letters }\end{array}$ \\
\hline & \multicolumn{6}{|c|}{ Cereal } \\
\hline Use By & $\begin{array}{c}38.75 \\
34.90,42.60\end{array}$ & A & $\begin{array}{c}2.58 \\
2.44,2.72\end{array}$ & & $\begin{array}{c}0.627 \\
0.546,0.707\end{array}$ & $\mathrm{~F}$ \\
\hline Fresh By & $\begin{array}{c}44.51 \\
40.84,48.18\end{array}$ & $\mathrm{BC}$ & $\begin{array}{c}2.10 \\
1.97,2.24\end{array}$ & $\mathrm{DE}$ & $\begin{array}{c}0.717 \\
0.641,0.794\end{array}$ & FG \\
\hline Best By & $\begin{array}{c}46.52 \\
43.49,49.55\end{array}$ & CD & $\begin{array}{c}1.96 \\
1.85,2.067\end{array}$ & CD & $\begin{array}{c}0.461 \\
0.398,0.524\end{array}$ & $\mathrm{E}$ \\
\hline \multirow[t]{2}{*}{ Sell By } & $\begin{array}{c}41.97 \\
38.41,45.52 \\
\end{array}$ & $\mathrm{ABC}$ & $\begin{array}{c}2.24 \\
2.11,2.36 \\
\end{array}$ & E & $\begin{array}{c}0.451 \\
0.376,0.525 \\
\end{array}$ & $\mathrm{E}$ \\
\hline & \multicolumn{6}{|c|}{ Salad } \\
\hline Use By & $\begin{array}{c}49.93 \\
46.09,53.77\end{array}$ & $\mathrm{D}$ & $\begin{array}{c}1.81 \\
1.67,1.95\end{array}$ & $\mathrm{C}$ & $\begin{array}{c}0.742 \\
0.662,0.823\end{array}$ & G \\
\hline Fresh By & $\begin{array}{c}45.53 \\
41.86,49.20\end{array}$ & CD & $\begin{array}{c}1.29 \\
1.16,1.42\end{array}$ & $\mathrm{AB}$ & $\begin{array}{c}0.374 \\
0.297,0.451\end{array}$ & $\mathrm{CDE}$ \\
\hline Best By & $\begin{array}{c}49.82 \\
46.79,52.85\end{array}$ & $\mathrm{D}$ & $\begin{array}{c}1.19 \\
1.080,1.30\end{array}$ & A & $\begin{array}{c}0.339 \\
0.275,0.402\end{array}$ & BCD \\
\hline \multirow[t]{2}{*}{ Sell By } & $\begin{array}{c}50.14 \\
46.58,53.69\end{array}$ & $\mathrm{D}$ & $\begin{array}{c}1.16 \\
1.034,1.29\end{array}$ & A & $\begin{array}{c}0.255 \\
0.180,0.329\end{array}$ & $\mathrm{AB}$ \\
\hline & \multicolumn{6}{|c|}{ Yogurt } \\
\hline Use By & $\begin{array}{c}37.52 \\
33.67,41.37\end{array}$ & A & $\begin{array}{c}1.46 \\
1.32,1.60\end{array}$ & B & $\begin{array}{c}0.429 \\
0.348,0.509\end{array}$ & $\mathrm{DE}$ \\
\hline Fresh By & $\begin{array}{c}37.73 \\
34.06,41.40\end{array}$ & A & $\begin{array}{c}1.33 \\
1.19,1.46\end{array}$ & $\mathrm{AB}$ & $\begin{array}{c}0.295 \\
0.218,0.372\end{array}$ & $\mathrm{ABC}$ \\
\hline Best By & $\begin{array}{c}40.47 \\
37.43,43.50\end{array}$ & $\mathrm{AB}$ & $\begin{array}{c}1.17 \\
1.056,1.28\end{array}$ & A & $\begin{array}{c}0.234 \\
0.170,0.297\end{array}$ & A \\
\hline Sell By & $\begin{array}{c}42.13 \\
38.57,45.68\end{array}$ & $\mathrm{ABC}$ & $\begin{array}{c}1.17 \\
1.045,1.30\end{array}$ & A & $\begin{array}{c}0.189 \\
0.115,0.264\end{array}$ & A \\
\hline
\end{tabular}

Note: Margins sharing a letter in the group label are not significantly different at the $5 \%$ level. The connecting letters are relevant down columns for PW, WTP, or WTW separately. The ANOVA contrasts are not corrected for multiplicity and empty cells are rebalanced. 


\section{References}

Buzby, J. C., Wells, H., F., \& Hyman, J. (2014). The Estimated Amount, Value, and Calories of Postharvest Food Losses at the Retail and Consumer Levels in the United States. In E. R. Service. Washington, D.C.: U.S. Department of Agriculture, Economic Research Service.

Codex Alimentarius Commission. (2014). Report of the Forty-Second Session of the Codex Committee on Food Labeling. In. Rome, Italy: Food and Agriculture Organization of the United Nations and World Health Oganization.

Coleman-Jensen, A., Rabbitt, M. P., Gregory, C., \& Singh, A. (2015). Household Food Security in the United States in 2014. In E. R. S. U.S. Department of Agriuclture. Washington, DC.

Ellsberg, D. (1961). Risk, ambiguity, and the Savage axioms. Quarterly Journal of Economics, 75, 643-669.

Farr-Wharton, G., Foth, M., \& Choi, J. H.-J. (2014). Identifying Factors that Promote Consumer Behaviours Causing Expired Domestic Food Waste. Journal of Consumer Behaviour, 13(6), 393-402.

Fox, C. R., \& Tversky, A. (1995). Ambiguity Aversion and Comparative Ignorance. Quarterly Journal of Economics, 110(3), 585-603.

Godfray, H. C. J., Beddington, J. R., Crute, I. R., Haddad, L., Lawrence, D., Muir, J. F., et al. (2010). Food Security: The Challenge of Feeding 9 Billion People. Science, 327(5967), 812-818.

Goldberg, I., Roosen, J., \& Nayga, R. M., Jr. (2009). Parental Response to Health Risk Information: Experimental Results on Willingness-to-Pay for Safer Infant Milk Formula. Health Economics, 18(5), 503-518.

Halloran, A., Clement, J., Kornum, N., Bucatariu, C., \& Magid, J. (2014). Addressing Food Waste Reduction in Denmark. Food Policy, 49, 294-301.

Kahneman, D. (2011). Thinking Fast and Slow. New York, New York: Farrar, Straus and Giroux.

Kahneman, D., \& Tversky, A. (1979). Intuitive Prediction: Biases and Corrective Procedures. TIMS Studies in Management Science, 12, 313-327.

Kantor, L. S., \& Lipton, K. (1997). Estimating and Addressing America's Food Losses. FoodReview, 20(1), 2.

Kivi, P. A., \& Shogren, J. F. (2010). Second-Order Ambiguity in Very Low Probability Risks: Food Safety Valuation. Journal of Agricultural and Resource Economics, 35(3), 443-456.

Leib, E. B., Gunders, D., Ferro, J., Nielsen, A., Nosek, G., \& Qu, J. (2013). The Dating Game: How Confusing Food Date Labels Lead to Food Waste in America. In, NRDC Report: Natural Resource Defense Council.

Lusk, J. L., Fields, D., \& Prevatt, W. (2008). An Incentive Compatible Conjoint Ranking Mechanism. American Journal of Agricultural Economics, 90(2), 487-498.

Lusk, J. L., \& Hudson, D. (2004). Willingness-to-pay estimates and their relevance to agribusiness decision making. Review of Agricultural Economics, 26(2), 152-169.

Miles, S., \& Frewer, L. J. (2001). Investigating Specific Concerns about Different Food Hazards. Food Quality and Preference, 12(1), 47-61.

Neff, R.A., Spiker, M.L., \& Truant, P.L. (2015). Wasted Food: U.S. Consumers’ Reported Awareness, Attitudes, and Behaviors. PLOS ONE 10(6).

DOI:10.1371/journla.pone.0127881. 
Newsome, R., Balestrini, C. G., Baum, M. D., Corby, J., Fisher, W., Goodburn, K., et al. (2014). Applications and Perceptions of Date Labeling of Food. Comprehensive Reviews in Food Science and Food Safety, 13(4), 745-769.

Pierson, T. R., Allen, J. W., McLaughlin, E. W., \& Halloran, J. M. (1982). Food Losses: Overview and Summary. Agricultural Economics Report 421 and Michigan Agricultural Experiment Station Journal Article 10876: Michigan Agricultural Experiment Station.

Theotokis, A., Pramatari, K., \& Tsiros, M. (2012). Effects of Expiration Date-Based Pricing on Brand Image Perceptions. Journal of Retailing, 88(1), 72-87.

Tsiros, M., \& Heilman, C. M. (2005). The Effect of Expiration Dates and Perceived Risk on Purchasing Behavior in Grocery Store Perishable Categories. Journal of Marketing, 69(2), 114-129.

Wansink, B., \& Wright, A. O. (2006). 'Best if Used By ...' How Freshness Dating Influences Food Acceptance. Journal of Food Science, 71(4), S354-S357.

Woodburn, M. J., \& Van Garde, S. J. (1987). Food discard practices of householders. Journal of the American Dietetic Association, 87(3), 322.

WRAP (Waste \& Resources Action Programme). (2011). Consumer Insight: Date Labels and Storage Guidance. Accessed February 22, 2015.

http://www.wrap.org.uk/sites/files/wrap/Technical\%20report\%20dates.pdf 


\section{Appendix A: Screenshot of the Auction Procedure (from 12/10/2014)}

\section{Cornell University}

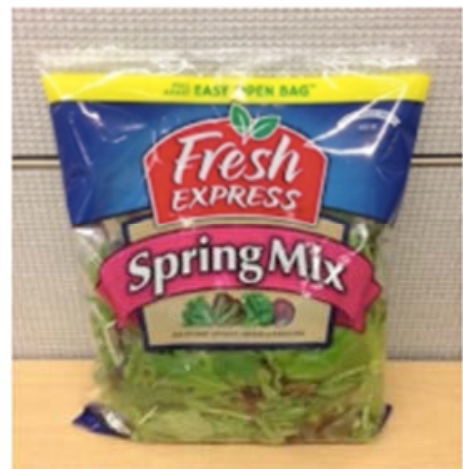

$5 \mathrm{oz}$. bag of washed salad greens

What percentage of the product are you (or your household) likely to consume, based on your recent consumption habits?

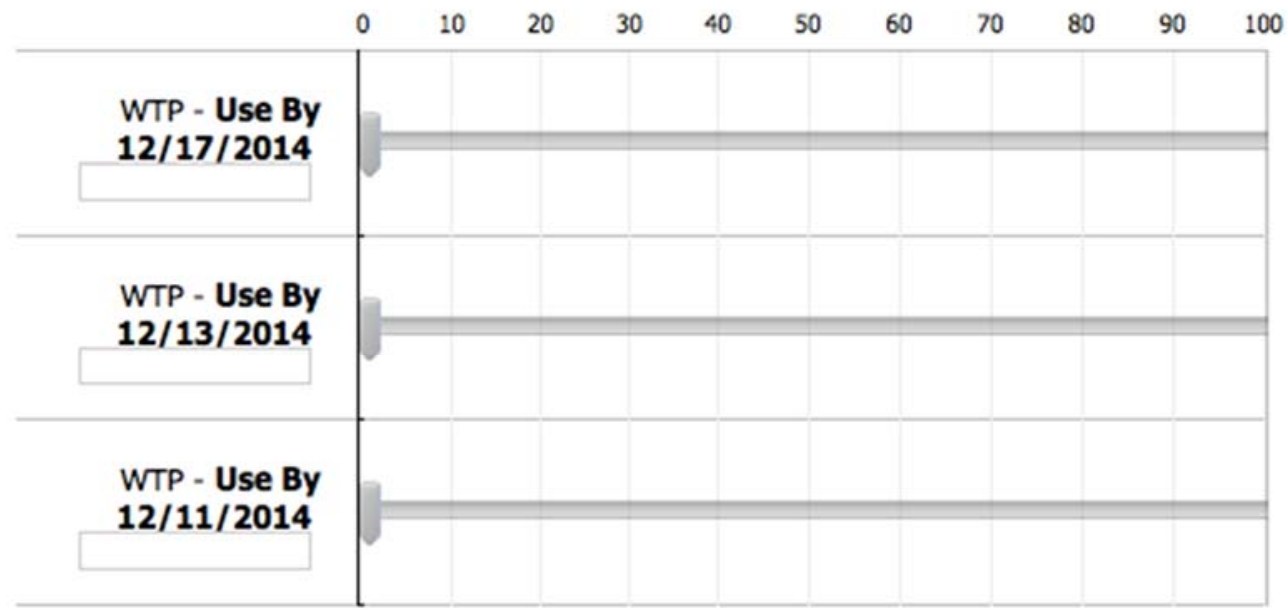

Note: The subjects were given clear directions to i) fill in their WTP for the product, and then ii)

to use the slider scale to show their likely level of consumption (between 0 and 100\%). 